\title{
Windowless, Silicon Nitride window and Polymer window EDS detectors: Changes in Sensitivity and Detectable Limits
}

\author{
J. Rafaelsen $^{1}$, T.Nylese ${ }^{1}$, M. Bolorizadeh ${ }^{1}$, V. Carlino ${ }^{2}$ \\ ${ }^{1}$ EDAX Inc, A division of Ametek, Materials Analysis Division, 91 McKee Drive, Mahwah, NJ \\ 07430 USA \\ 2ibss Group INC, 1559B Sloat Blvs., Suite 270, San Francisco, Ca 94132 USA
}

With the introduction of Silicon Drift Detectors (SDD) and the development of fast and low noise pulse processors, Energy Dispersive Spectroscopy (EDS) analysis has seen remarkable increases in throughput and reliability in the last decade. But one often overlooked aspect of the detection technology is the x-ray window. While windowless detectors are becoming the standard for Transmission Electron Microscopes (TEM), they are rarely used on Scanning Electron Microscopes (SEM), primarily due to the risk of detector contamination when venting the vacuum chamber. By adding a sealed window in front of the detector module it is possible to keep the detector cooled and under vacuum at all times, eliminating the risk of detector contamination during venting cycles. A variety of window technologies are available including Beryllium, polymer films, and the most recent addition, silicon nitride.

The different window materials will have an effect on the measured spectrum due to the x-ray absorption in the window, but also the support grid for the window influences the spectra. Figure 1 shows an SEM image of the window structure for silicon nitride and polymer windows. The hexagonal pattern of the support grid for the silicon nitride window covers roughly $18 \%$ of the total area while the venetian blind support structure for the polymer window covers roughly $23 \%$ of the total area.

In this work, we characterize the differences between silicon nitride and polymer windows and compare those to the performance of a windowless detector. To this end we have collected a series of spectra from a sample block containing traceable standards using a windowless detector. We then mounted a cap in front of the electron trap containing either a silicon nitride or polymer window. By using a windowless detector and exchanging windows, we can use the same detector for all measurements and thus ensure that the results are not affected by detector characteristics. Though the addition of a cap containing a window will work as a limiting aperture and reduce the solid angle of the detector, comparable statics between windows were ensured by acquiring the same number of counts in the spectra. An example of the spectra can be seen in Figure 2.

In order to quantify the differences between the windows, we calculated the Minimum Detection Limit (MDL) for spectra acquired from the same samples using the three different detector/window configurations. While there are several models for calculating the MDL (1) (2), we applied the criteria the peak height should be higher than the background with a $95.45 \%$ confidence level.

Plasma cleaners have become common accessories to modern SEMs as they help reduce sample and chamber contamination, and they have even been shown to improve light element sensitivity in EDS measurements (3). However, there is still debate about to which extent cleaning is compatible with $\mathrm{x}$-ray windows. We will present, to our knowledge, the first comparison 
between plasma cleaning of polymer and silicon nitride windows. The plasma cleaning experiments were conducted with a GV10x Asher from ibss Group Inc. in a dedicated chamber.

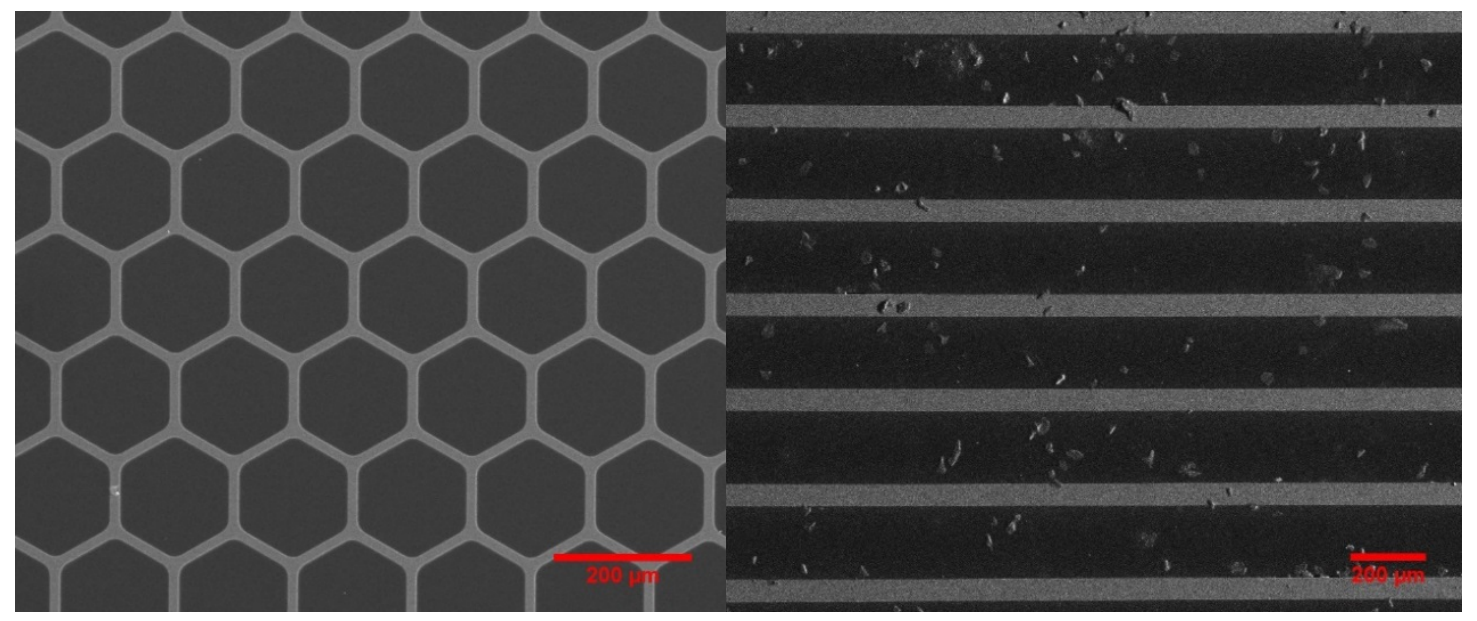

Figure 1: SEM images showing the support grids for silicon nitride window (left) and polymer window (right).

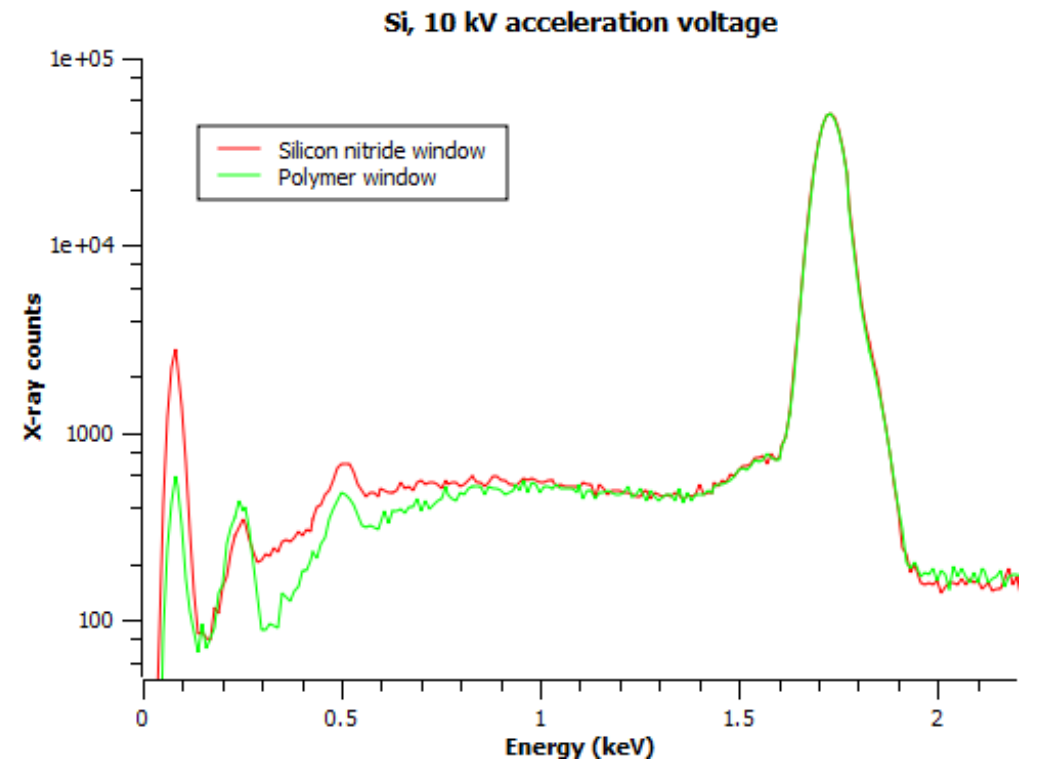

Figure 2: Si spectra for polymer and silicon nitride window.

\section{References}

1. Precision and Sensitivity in Electron Microprobe Analysis. Ziebold, Thomas O. 8, 1967, Analytical Chemistry, Vol. 39, pp. 858-861.

2. Goldstein, et al. Scanning Electron Microscopy and X-Ray Microanalysis. s.l. : Plenum Press, New York, 1984. ISBN 0-306-40768-X.

3. Improved Carbon Analysis with Evactron Plasma Cleaning. Rolland, P., Carlino, V. and Vane, R. . S02, s.l. : Microscopy Society of America, 2004, Microscopy and Microanalysis, Vol. 10, pp. 964-965x. 